the quality of important research. If some people oppose participation in biobank research very strongly, this makes their inclusion worse, but then they are most likely motivated to communicate this to researchers or biobank holders, and when they do, the opt-out door is wide open. It is hard to see that such a practice would be detrimental to people's trust in medical research.

1. Helgesson, G., Dillner, J., Carlson, J., Bartram, C.R. \& Hansson, M.G. Nat. Biotechnol. 25, 973-976 (2007).

2. Eriksson, S. \& Helgesson, G. Eur. J. Hum. Genet. 13, 1071-1076 (2005).

3. http://www.wma.net/e/policy/b3.htm

4. Hansson, M.G., Dillner, J, Bartram, C.R., Carlsson, J. \& Helgesson, G. Lancet Oncol. 3, 266-269 (2006).

\title{
Cost/success projections for US biodefense countermeasure development
}

\section{To the Editor:}

To protect civilians against biological weapons and bioterrorism, the US Department of Health and Human Services (HHS) has developed a list of essential medical countermeasure requirements. We performed a survey of candidate biodefense countermeasures in development and estimated their future clinical development costs, based on historical drug and vaccine development data. The cost of supporting existing candidates through clinical development is estimated to be $\$ 4.1$ billion over the next seven years, with costs of $\$ 817$ million in fiscal year 2009, alone. Given the high failure rate of biopharmaceutical development, the probability of developing approved products from the existing pipeline is between $12 \%$ and $85 \%$ per HHS requirement. To increase the probability to $90 \%$, two to nine additional candidates will be needed per requirement, at a total seven-year clinical development cost of $\$ 14.0$ billion. To date, the primary government program tasked with supporting clinical development of medical countermeasures has received only \$201 million.

In 2006, the Pandemic and All-hazards Preparedness Act (PAHPA, PL 109-417) was signed into law and created the Biomedical Advanced Research and Development Authority (BARDA) within the HHS. One of BARDA's core missions, as defined by PAHPA, is to promote the clinical development of medical countermeasures (MCMs) including drugs and vaccines_-effective against chemical, biological, radiological and nuclear (CBRN) threats to the United States ${ }^{1}$. BARDA was intended to bridge the 'valley of death' between National Institute of Allergy and Infectious Diseases (NIAID) funding for basic research and early preclinical development, and procurement of successfully developed products via the BioShield program that was established by law in 2004 (P.L. 108-

276) (Supplementary Fig. 1 online).

In the April 2007 Public Health Emergency Medical Countermeasure Enterprise
Table 1 Probability of existing candidates satisfying HHS requirements

\begin{tabular}{|c|c|c|c|c|c|c|c|}
\hline \multirow[b]{2}{*}{ HHS requirement } & \multicolumn{3}{|c|}{ Number of candidates } & \multicolumn{3}{|c|}{$\begin{array}{c}\text { Probability of eventual } \\
\text { approval }^{\mathrm{a}}\end{array}$} & \multirow{2}{*}{$\begin{array}{l}\text { Probability of } \\
\text { at least one } \\
\text { approval per } \\
\text { requirement }\end{array}$} \\
\hline & Preclinical & Phase 1 & Phase 2 & Preclinical & Phase 1 & Phase 2 & \\
\hline Anthrax vaccine & 6 & 3 & 1 & $12 \%$ & $21 \%$ & $30 \%$ & $85 \%$ \\
\hline Anthrax antitoxin & 3 & 2 & 0 & $17 \%$ & $30 \%$ & $36 \%$ & $72 \%$ \\
\hline Filovirus vaccine & 2 & 3 & 0 & $12 \%$ & $21 \%$ & $30 \%$ & $63 \%$ \\
\hline Filovirus antiviral & 4 & 0 & 0 & $12 \%$ & $21 \%$ & $30 \%$ & $41 \%$ \\
\hline Junin virus antiviral & 2 & 0 & 0 & $12 \%$ & $21 \%$ & $30 \%$ & $23 \%$ \\
\hline Smallpox antiviral & 2 & 2 & 0 & $12 \%$ & $21 \%$ & $30 \%$ & $53 \%$ \\
\hline $\begin{array}{l}\text { Broad-spectrum antibiotic } \\
\text { against Gram-positives }\end{array}$ & 1 & 0 & 0 & $12 \%$ & $21 \%$ & $30 \%$ & $12 \%$ \\
\hline $\begin{array}{l}\text { Broad-spectrum antibiotic } \\
\text { against Gram-negatives }\end{array}$ & 1 & 0 & 0 & $12 \%$ & $21 \%$ & $30 \%$ & $12 \%$ \\
\hline
\end{tabular}

aThese estimates are based on historical, aggregate data, and are not meant to reflect judgments of the merits of any specific product currently in development.
(PHEMCE) Implementation Plan ${ }^{1}$, HHS defined a series of requirements for MCMs deemed essential to protect civilians against CBRN threats. Currently, several of these MCMs are under development and are in, or have the potential to soon enter, clinical development, making them eligible for funding from BARDA. Since its creation, BARDA has received a total of \$201 million from the US Congress, in fiscal years (FY) 2007 and 2008 (refs. 2,3). However, to date, there has been no estimate of the actual costs of developing these MCMs, or of the probability of successful licensure given current and potential future funding levels.

We surveyed the pipeline of MCM candidates in or entering clinical development that are responsive to the eight HHS biodefense MCM requirements described in the HHS PHEMCE Implementation Plan $^{1}$ (Supplementary Box 1 online and Table 1). Candidates were identified from pharmaceutical and biotech companies' press releases and quarterly and/or annual reports, news reports, US government agency reports and databases (http://www3.niaid.nih.gov/ topics/BiodefenseRelated/Biodefense/research/ funding/default.htm; http://clinicaltrials.gov/), a 2006 biodefense market survey ${ }^{4}$ and through discussions with HHS staff and members of the Alliance for Biosecurity (http://www. allianceforbiosecurity.org)—a collaboration between the Center for Biosecurity of the University of Pittsburgh Medical Center and 13 biopharmaceutical companies. Our analysis included only biodefense drug and vaccine requirements and thus excluded countermeasures against radiological, nuclear or chemical weapons, as well as diagnostics and biodosimetry assays.

Mean development out-of-pocket costs, durations and development phase transition probabilities were based on historical data (Supplementary Methods online). Candidate MCMs in clinical development in FY2009 were projected by assuming that candidate MCMs now in preclinical development will be in phase 1 trials with probability equal to the average preclinical-phase 1 transition probability; those now in phase 1 trials will enter phase 2 trials with probability equal to the average phase 1-phase 2 transition probability; and those now in phase 2 trials will, assuming they are now mid-phase, remain in their current phase for all of FY2009 (Supplementary Table 2 online).

Total costs of clinical development for existing candidate MCMs were calculated by multiplying the number of candidate MCMs projected to be in each clinical phase, by the respective costs per remaining phases and 
those phases' respective transition probabilities. FY2009 costs of clinical development for existing candidate MCMs were calculated by multiplying the number of candidate MCMs projected to be in each clinical phase, by the respective annual cost per FY2009 phase. The probability of at least one approved product per requirement was calculated as:

$$
1-\prod_{i}[1-\operatorname{Pr}\langle\text { Approval } \mid i\rangle]^{N i}
$$

where $\mathrm{Ni}$ is the number of candidates at stage i. These probabilities are based on historical, aggregate data and are not judgments of the merits of particular products. BARDA documentation does not set targets for the probability of US Food and Drug Administration (Rockville, MD) approval. We estimated costs for approval probabilities of $70 \%, 80 \%$ and $90 \%$.

Our January 2008 survey identified 32 candidates that could fulfill HHS biodefense MCM requirements, including 12 small-

을 molecule drugs, 15 vaccines and 5 biological therapeutics (Supplementary Table 1 online). Of these candidates, 21 are, as of this writing, in preclinical development, 10 are in phase 1 trials, and one is in phase 2 trials. Given expected costs, timelines and success rates (Supplementary Table 2), the direct (out-ofpocket) costs for clinical development of all existing candidates up to failure or approval would total \$4,097 million over the next seven years - the mean duration of clinical development. Costs for FY2009, alone, would total \$817 million (Supplementary Table 3 online).

On the basis of historical pharmaceutical failure rates, the probability of at least one approved product per HHS requirement within the existing pipeline is $12 \%$ for the broad-spectrum antibiotics, $23 \%$ for a Junin virus antiviral, $41 \%$ for a filovirus antiviral, $53 \%$ for a smallpox antiviral, $63 \%$ for a filovirus vaccine, $72 \%$ for an anthrax antitoxin and $85 \%$ for an anthrax vaccine (Table 1).

To yield at least a $90 \%$ probability of one approved product for each of the eight HHS biodefense requirements identified in this study, the pipeline of candidate MCMs will need to be expanded by two to nine additional candidates per requirement (Table 2).

Supporting the clinical development of these additional candidates up to failure or approval would cost $\sim \$ 9.9$ billion over seven years. Adding those costs to the clinical development costs of existing candidates, the clinical development costs for a pipeline large enough to have a $90 \%$ probability of yielding at least one approved MCM per HHS requirement

Table 2 Additional candidates needed to increase probability of satisfying HHS requirements

Additional phase 1 candidates needed for one approved product per requirement with probability

\begin{tabular}{lccc} 
& $\mathbf{7 0} \%$ & $\mathbf{8 0} \%$ & $\mathbf{9 0 \%}$ \\
\hline HHS requirement & 0 & 0 & 2 \\
\hline Anthrax vaccine & 0 & 1 & 3 \\
\hline Anthrax antitoxin & 1 & 3 & 6 \\
\hline Filovirus vaccine & 3 & 5 & 8 \\
\hline Filovirus antiviral & 4 & 6 & 9 \\
\hline Junin virus antiviral & 2 & 4 & 7 \\
Smallpox antiviral & 5 & 7 & 9 \\
Broad-spectrum antibiotic against Gram-positives & 5 & 7 & 9 \\
\hline Broad-spectrum antibiotic against Gram-negatives & 20 & 33 & 53 \\
\hline Total & 992 & 1,168 & 2,573 \\
\hline $\begin{array}{l}\text { FYO9 clinical development costs for the additional } \\
\text { candidate MCMs (\$ millions, 2008) }\end{array}$ & & 2,436 & 3,390 \\
$\begin{array}{l}\text { FY09 clinical development costs for existing pipeline } \\
\text { plus additional candidate MCMs (\$ millions, 2008) }\end{array}$ & 1,809 & 10,292 & 14,016 \\
$\begin{array}{l}\text { Total clinical development costs for existing pipeline } \\
\text { plus additional candidate MCMs, FY2009-2015 }\end{array}$ & 7,864 & & \\
(\$ millions, 2008) & & &
\end{tabular}

would thus be $\$ 14.0$ billion over seven years, and $\$ 3.39$ billion in FY2009.

Our estimates for BARDA's funding needs are significantly higher than the $\$ 102$ million appropriated by the Congress for FY2008 and the $\$ 250$ million requested in the President's FY2009 budget. In fact, one year of $\$ 102$ million in funding to BARDA is sufficient to support only two candidate MCMs for one year; and based on historical success rates, each of those candidates would have at most a $30 \%$ probability of eventually gaining FDA approval.

Our analysis illustrates that, based on historical data of biopharmaceutical development, funding allocations for clinical development of biodefense MCMs have direct impacts on the probability of successfully satisfying PHEMCE requirements. The US government, including the White House, HHS and Congress, must decide what probability of failure it is willing to tolerate, and make the associated commitment to MCM development. On August 1, 2008, the White House submitted a request to Congress to amend the President's FY2009 budget request for BARDA's MCM advanced development mission from $\$ 250$ million to $\$ 723$ million. As of this writing, Congress has not finalized the BARDA FY2009 appropriation. If funding does not significantly increase, HHS cannot respond to all of the biological agents currently designated by the Department of Homeland Security as "material threats" (and subsequently listed as requirements in the PHEMCE Implementation Plan).

In addition to increasing funding, we suggest that indirect economic incentives for biodefense MCM development, such as priority review vouchers, should be created to further promote MCM development ${ }^{5,6}$. In 2007, legislation was passed to provide priority review vouchers to firms that develop pharmaceuticals targeting diseases that predominantly affect developing countries ${ }^{7}$. This incentive could also be expanded to include biodefense countermeasures, at no cost to taxpayers 5 .

Absent appropriate funding, BARDA could consider focusing its limited funds on the one or two biological agents believed to present the greatest risk. Alternatively, BARDA could shift some of its funding from agent-specific countermeasures to more "flexible defenses" that address multiple pathogens, such as broadspectrum countermeasures and platform technologies (e.g., vaccine adjuvants and delivery systems and MCM rapid development and production technologies). Although flexible defenses may take longer to develop and have higher technical risk, they might prove more cost effective in the long run ${ }^{8}$.

Note: Supplementary information is available on the Nature Biotechnology website.

\section{ACKNOWLEDGMENTS}

The authors are grateful to Luciana Borio, Gigi Gronvall, Dawn Myscofski and four anonymous reviewers for comments on an earlier draft.

\section{Jason Matheny ${ }^{1,2}$, Michael Mair ${ }^{1}$ \& Bradley Smith $^{1}$}

${ }^{1}$ Center for Biosecurity of the University of Pittsburgh Medical Center, 621 E. Pratt Street, Suite 210, Baltimore, Maryland 21202, USA. 
${ }^{2}$ Johns Hopkins Bloomberg School of Public Health, Department of Health Policy and Management, 624 North Broadway, Baltimore, Maryland 21205, USA.

e-mail:jmatheny@jhsph.edu

1. US Department of Health and Human Services. Public Health Emergency Medical Countermeasure Enterprise, Implementation Plan for Chemical, Biological, Radiological, and Nuclear Threats. April 2007. <http://www.hhs.gov/ aspr/barda/documents/phemce_implplan_041607final. pdf >; accessed August 22, 2008.

2. Office of Legislative Policy and Analysis. Public Law 110-28 (H.R. 2206) <http://olpa.od.nih.gov/ legislation/110/publiclaws/supplementalFY2007.asp>; accessed January 30, 2008.
3. $110^{\text {th }}$ Congress of the USA. Consolidated Appropriations Act, 2008. Public Law 110-161, pp. 341, 369; $<$ http://frwebgate.access.gpo.gov/cgi-bin/getdoc. cgi?dbname $=110$ cong_bills\&docid $=f$ :h2764enr.txt. pdf $>$; accessed January 30, 2008.

4. Trull, M.C., du Laney, T.V. \& Dibner, M.D. Nat. Biotechnol. 25, 179-184 (2007)

5. Matheny, J.G., Mair, M., Mulcahy, A. \& Smith, B.T. Biosecur. Bioterror. 5, 228-238 (2007).

6. Ridley, D.B., Grabowski, H.G. \& Moe, J.L. Health Aff. (Millwood) 25, 313-324 (2006).

7. $110^{\text {th }}$ Congress of the USA. Public Law 110-85 (H.R. 3580); <http://frwebgate.access.gpo.gov/ cgi-bin/getdoc.cgi?dbname=110_cong_public_ laws\&docid=f:publ085.110>; accessed June 2, 2008.

8. Gronvall, G.K. et al. Biosecur. Bioterror. 5, 271-277 (2007).

\section{Data completeness-the Achilles heel of drug-target networks}

\section{To the Editor:}

The use of network-based approaches to visualize and analyze different types of 을 biologically relevant interaction data has become increasingly popular in recent years. Topological studies of these interaction networks offer a means to assess the interconnectivity structure established among and between diseases, genes, proteins, and molecules from which influential conclusions and global trends in biology and drug discovery can be derived ${ }^{1-3}$. However, in spite of its indubitable value, currently available interaction data is far from being complete and the portion accessible is often nonhomogeneous and biased toward certain areas of interest ${ }^{4}$. This situation results in sample networks that may not be representative of the whole network and thus caution on the conclusions drawn should be highlighted.

Here, we are particularly interested in assessing current levels of completeness in available drug-target interaction data, the potential implications for the topology of the networks derived from them and the impact that changes in network topology may have on the view of the current status of drug discovery. In this respect, it is widely recognized that, due to limited time and resources, small molecules are usually not screened systematically through a large panel of protein targets for the sake of acquiring knowledge about their complete pharmacological profile but solely to the few targets of interest for the particular project at work. The consequences are that the drug-target interaction data currently available from public bibliographic sources and stored in annotated chemical libraries are largely incomplete and biased toward targets of common therapeutic interest. For example, although the typical antipsychotic drug chlorpromazine is connected in DrugBank ${ }^{5}$ to two primary aminergic $G$ protein-coupled receptor (aGPCR) targets (namely, dopamine $\mathrm{D}_{2}$ and serotonin $5-\mathrm{HT}_{2 \mathrm{~A}}$ ), and in Wombat ${ }^{6}$ is annotated to another three aGPCRs (namely, dopamine $\mathrm{D}_{1}$ and $\mathrm{D}_{3}$, and serotonin $5-\mathrm{HT}_{1 \mathrm{~A}}$ ), a more complete 'receptorome' profiling of this drug$^{7}$ shows that it actually has submicromolar affinity for at least 19 additional aGPCRs.

To gain a deeper insight on the effect that data completeness may have on the topology of drug-target interaction networks, we took a set of 829 approved small-molecule drugs from DrugBank and complemented systematically the original interaction data, first, with additional literature-based experimental data available in Wombat and, second, with estimated data obtained from an in silico target profiling method. On the basis of the three sets of interaction data compiled with increased levels of completeness, we constructed drugtarget networks, in which a drug and a protein are connected to each other if the protein is a known target of the drug (Fig. 1). We then compared the topology of the three drug-target networks in terms of the fraction of nodes belonging to the largest connected component (nLCC) in the corresponding drug network, in which nodes are drugs connected if they share at least one target, and target network, in which nodes are targets connected if they share at least one drug.

The first drug-target network was thus based on the 1,445 drug-target interactions available in DrugBank connecting the 829 drugs to 314 protein targets (Fig. 1a), resulting in an average number of target proteins per drug of
1.7. The topology of this drug-target network reveals a well-organized modular structure, with many proteins naturally clustering around phylogenetic families. The nLCC values for the corresponding drug and target networks are 0.60 and 0.42 , respectively. Both these numbers are significantly smaller than the values of 0.90 and 0.78 obtained from randomizing the networks while keeping the number of nodes and links unchanged. The two nLCC values are also found very much in agreement with those reported recently from networks derived using the same data ${ }^{8}$. However, because DrugBank contains mainly information on the primary targets of drugs (that is, those proteins formally accepted by the originators as being targeted by drugs), its interaction data suffer from the incompleteness and bias issues emphasized above $^{9}$. In fact, a drug-target network derived solely on the basis of DrugBank interaction data may actually be more representative of the target space explored historically by the pharmaceutical industry rather than being a true reflection of drug polypharmacology.

Accordingly, we constructed a second drugtarget network by supplementing the original interaction data in DrugBank with additional literature-based affinity data available in Wombat, which resulted in 2,117 drug-target interactions connecting the 829 drugs to 409 protein targets (Fig. 1b). This means that the networks derived from these data account for 672 extra drug-target interactions and 95 proteins relative to the original data present in DrugBank, increasing the average number of target proteins per drug to 2.7. The topology of the drug-target network is visibly affected, becoming more complex and interconnected. Quantitatively, this is reflected by nLCC values of 0.79 and 0.64 for the corresponding drug and target networks, respectively; these values are much closer to the topology of a randomized network than those resulting from a network constructed from DrugBank data alone.

We derived a final third drug-target network by complementing the literaturebased experimental data accumulated from DrugBank and Wombat with annotations assigned using a ligand-based approach to in silico target profiling recently reported by our group ${ }^{10}$. This new network consists of a total of 5,215 drug-target interactions connecting the 829 drugs to 557 targets (Fig. 1c). On the basis of the network, the average number of target proteins per drug increases to 6.3. Even though the number of drug-target interactions obtained at this stage might seem quite large compared with the previous two cases that considered literature-based experimental data alone, the projection obtained agrees well 